\title{
Acute miliary tuberculosis in pregnancy after in vitro fertilization and embryo transfer: a report of seven cases
}

\author{
Xiaoyan Gai ${ }^{1 \dagger}$, Hongbin Chi ${ }^{2,5,6,7 \dagger}$, Wenli Cao ${ }^{3 \dagger}$, Lin Zeng ${ }^{4}$, Lixue Chen ${ }^{2,5,6,7}$, Weixia Zhang ${ }^{1}$, \\ Donghong Song ${ }^{2,5,6,7}$, Ying Wang ${ }^{2,5,6,7}$, Ping Liu ${ }^{2,5,6,7}$, Rong $\mathrm{Li}^{2,5,6,7^{*}}$ and Yongchang Sun ${ }^{{ }^{*}}$
}

\begin{abstract}
Background: While miliary tuberculosis (TB) in pregnancy is rare after in vitro fertilization and embryo transfer (IVFET), it poses a serious threat to the health of pregnant women and their fetuses. The present study aimed to describe the clinical features of miliary TB and pregnancy outcomes of patients after IVF-ET.

Methods: Data of infertile patients who received IVF-ET at Peking University Third Hospital between January 2012 to December 2017 were retrospectively analyzed. Patients who developed miliary TB during pregnancy were identified, and clinical characteristics of miliary TB were described.

Results: Out of 62,755 infertile women enrolled, 7137 (11.4\%) showed signs of prior pulmonary TB on chest X-ray (CXR). Among the 15,136 women (mean age: $33.2 \pm 5.0$ years) who successfully achieved clinical pregnancy, seven patients aged 28-35 years had miliary TB during pregnancy, with two patients having a complication of TB meningitis. All these patients presented with fever. Notably, old TB lesions were detected on CXR in six patients before IVF-ET; nevertheless, no anti-TB therapy was administered. Furthermore, salpingography revealed oviduct obstruction in all patients (7/7). Patients received anti-TB therapy following a diagnosis of miliary TB and were clinically cured. However, pregnancy was terminated due to spontaneous (4/7) and induced (3/7) abortion.
\end{abstract}

Conclusions: TB reactivation, mostly as miliary TB and TB meningitis, is severe in pregnant women after IVF-ET and deleterious to pregnancy outcomes. Signs of prior TB on CXR may be risk factors for TB reactivation during pregnancy.

Keywords: Miliary tuberculosis, Infertility, In vitro fertilization, Embryo transfer

\section{Background}

Tuberculosis (TB) remains a major public health problem globally and poses a considerable threat to human health [1]. Globally, approximately 3.2 million women

*Correspondence: roseli001@sina.com; suny@bjmu.edu.cn

${ }^{+}$Xiaoyan Gai,Hongbin Chi and Wenli Cao are joint first authors

${ }^{1}$ Department of Respiratory and Critical Care Medicine, Peking University

Third Hospital, 49 N Garden Rd, Haidian District, Beijing 100191, China

${ }^{2}$ Center for Reproductive Medicine, Department of Obstetrics

and Gynecology, Peking University Third Hospital, 49 N Garden Rd,

Haidian District, 100191 Beijing, China

Full list of author information is available at the end of the article suffer from clinical TB each year [2]. Pregnancy-related $\mathrm{TB}$ endangers the health of both women and their fetuses and is considered an important cause of morbidity and mortality [3-5]. TB more rapidly progresses in pregnant women than in nonpregnant ones [5] and can lead to miscarriage [3-5]. Furthermore, women who survived from TB are often dissuaded from having children or, even worse, can no longer conceive again.

Acute miliary TB, a more serious and potentially lethal form of the disease, results from massive hematogenous dissemination of Mycobacterium tuberculosis. The miliary pattern in the lungs has been radiologically described as 
"a collection of tiny discrete pulmonary opacities that are generally uniform in size and widespread in distribution, each of which measures $2 \mathrm{~mm}$ or less in diameter" [6, 7]. If untreated, miliary $\mathrm{TB}$ is uniformly fatal. Relative to all forms of TB, the incidence of miliary $\mathrm{TB}$ ranges from 0.15 to $10 \%[1,6,7]$. Additionally, approximately $15-30 \%$ of patients with pulmonary TB during pregnancy exhibit hematogenous dissemination and have miliary TB [8]. Because clinical symptoms such as fever and cough are nonspecific and chest X-ray (CXR) and chest computed tomography $(\mathrm{CT})$ scan during pregnancy are associated with a risk of radiation exposure, the diagnosis of miliary TB during pregnancy is often delayed.

With the increasing application of in vitro fertilization and embryo transfer (IVF-ET), the incidence of TB during pregnancy has gradually increased, posing a serious threat to the health of pregnant women and fetuses $[1,9$, 10]. There have been occasional case reports of TB with hematogenous dissemination, miliary TB, and/or meningitis during pregnancy after IVF-ET, leading to abortion, fetal malformation, or increased risk of mortality [10, 11 . Therefore, correct and timely diagnosis and management of TB during pregnancy are important. Therefore, in this study, we aimed to describe the clinical features of TB and its impact on pregnancy outcomes after IVFET. We retrospectively analyzed the data of patients who underwent IVF-ET and showed clinical signs of miliary TB during pregnancy between January 2012 and December 2017 at the reproductive center of our hospital. Additionally, we summarized the clinical manifestations and pregnancy outcomes of these patients.

\section{Methods}

This was a retrospective study of patients who underwent IVF-ET for infertility between January 1, 2012, and December 31, 2017, at Peking University Third Hospital, a tertiary referral hospital in Beijing, China. Data on patients undergoing IVF-ET, including causes of infertility, serum hormone concentrations, the controlled ovarian hyperstimulation protocol, and CXR results, were recorded. CXR was routinely performed for each patient, and active TB cases were excluded before IVF-ET was started. A medical team was assigned to follow up the pregnancy outcomes.

During the 6-year period, 62,755 patients, who were all HIV-negative, had received IVF-ET at our center. Among these patients, seven with active TB during pregnancy were identified. Active TB was diagnosed according to the national guidelines [12]. Miliary TB was diagnosed based on the size, distribution, and density of miliary-like nodules that were bilaterally distributed on CXR or chest CT scan $[13,14]$. Baseline data and CXR and laparoscopy results before IVF-ET were retrieved. A respiratory physician contacted the seven patients via phone call and reviewed the medical records. Live birth was defined as the delivery of a living fetus (or living fetuses) beyond 28 weeks of gestation, whereas miscarriage was defined as pregnancy loss before 28 weeks of gestation.

This study was approved by the Ethics Committee of Peking University Third Hospital [batch number: (2019)327-02]. The retrospective nature of the study resulted in a waiver regarding the signing of the informed consent form.

\section{IVF-ET protocol}

IVF-ET was performed as previously described [15]. Briefly, controlled ovarian hyperstimulation was achieved, oocytes were fertilized, and ETs were subsequently performed [15]. Among seven patients who developed TB during pregnancy, one had undergone a frozen cycle transfer, whereas the remaining six had undergone fresh cycle transfer. After ET, $60 \mathrm{mg}$ of progesterone was injected intramuscularly for 14 days. Blood human chorionic gonadotropin concentration was monitored at 2 weeks after transplantation, and the status of the embryo sac was examined by ultrasonography at 4 weeks after transplantation.

\section{Statistical analysis}

Continuous variables are expressed as mean \pm standard deviation or as median with interquartile range. Statistical analysis was performed using SPSS version 23 (IBM Corp., Armonk, NY).

\section{Results}

\section{Patients' baseline data}

A total of 62,755 infertile patients (mean age: $33.1 \pm 5.1$ years, range: $20-50$ years) were identified from our database to have been referred to the reproductive center of our hospital between January 1, 2012, and December 31, 2017. Of these patients, $11.4 \%(7137 / 62,755)$ exhibited signs of prior pulmonary TB prior to IVF-ET based on their CXR results. Furthermore, 37,854 out of all 62,755 patients underwent ET, whereas the remaining 24,901 patients failed because they had no ovum that could be obtained or had no embryo to transfer or due to some other reasons. Finally, 15,136 (mean age: $33.2 \pm 5.0$ years) out of 37,854 patients succeeded in achieving clinical pregnancy. Among these 15,136 patients, seven had acute miliary TB during pregnancy. Hence, the prevalence rate was $7 / 15,136$ (i.e., 4.6/10,000) (Fig. 1).

\section{Baseline data of the seven patients with active TB during pregnancy}

Among the seven cases, four occurred in 2012, two in 2016, and one in 2017. These pregnant women were 


\section{2,755 patients who underwent IVF-ET}

from January 2012 to December 2017

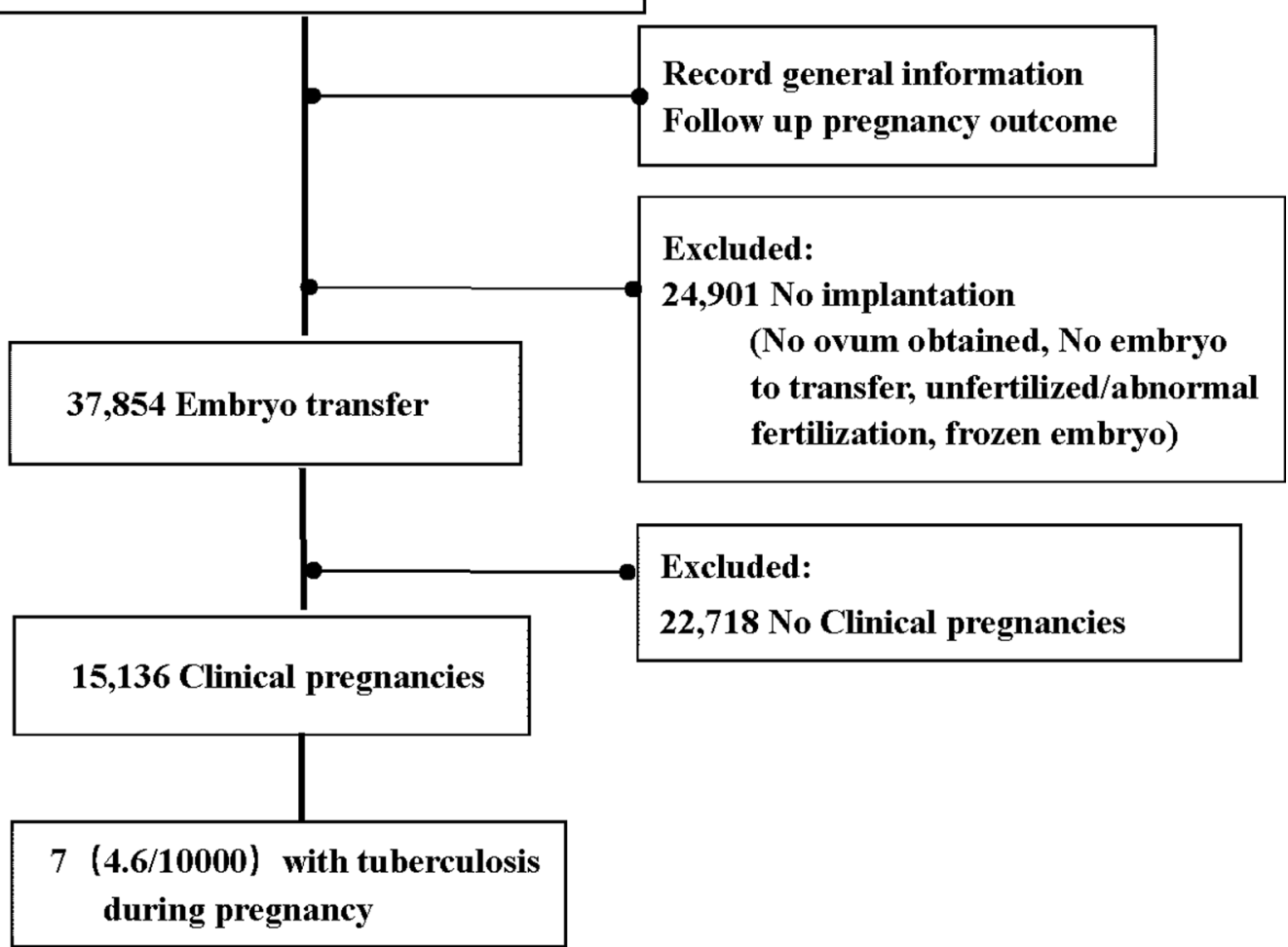

Fig. 1 Flow of protocol and outcomes

between 28 and 34 years of age, and the duration of infertility ranged from 1 to 12 years. Their body mass index was $20.1-27.3 \mathrm{~kg} / \mathrm{m}^{2}$. All seven patients had primary infertility due to unilateral or bilateral oviduct obstruction, as assessed using salpingography. Four patients also underwent laparoscopy and showed tubal obstruction and adhesion consistent with TB; however, the pathology failed to reveal features of TB. One patient had suffered from TB at the age of 16, and the local hospital administered anti-TB therapies for over 6 months at that time. The other six patients had no clinical history of $\mathrm{TB}$ and had not received anti-TB treatment. Among the seven patients, six showed signs of old pulmonary TB lesions on CXR before IVF-ET. Tuberculin skin test (TST) was performed in three patients before IVF-ET, with induration diameters of $10-20 \mathrm{~mm}$, thus confirmed as positive $(++)$ cases. However, this test was not performed in the other four patients. None had active TB before IVF-ET, and IVFET was performed as scheduled (Fig. 2; Table 1).

One patient underwent frozen ET and had a singleton pregnancy. The remaining six patients underwent fresh
ET: three had twin pregnancies and three had singleton pregnancies.

\section{Clinical manifestations and diagnosis of active TB during pregnancy}

All seven patients had fever at 7-14 weeks of pregnancy. Among them, six had moderate-to-high fever, with the highest body temperatures recorded at 38.5$40{ }^{\circ} \mathrm{C}$, whereas one had low-grade fever $\left(37.5^{\circ} \mathrm{C}\right)$. All seven patients had mild cough and a small amount of sputum with (1/7) or without blood (6/7). CXR and CT scans were performed on all seven patients, which showed diffuse miliary nodules in both lungs, consistent with acute miliary TB (Fig. 2). Two patients with complaints of significant headache were confirmed by lumbar puncture to have TB meningitis (Table 1).

Five patients underwent an interferon gamma release assay (IGRA) test after fever onset and showed positive results. One patient underwent the TST, which was positive $(+++)$. 

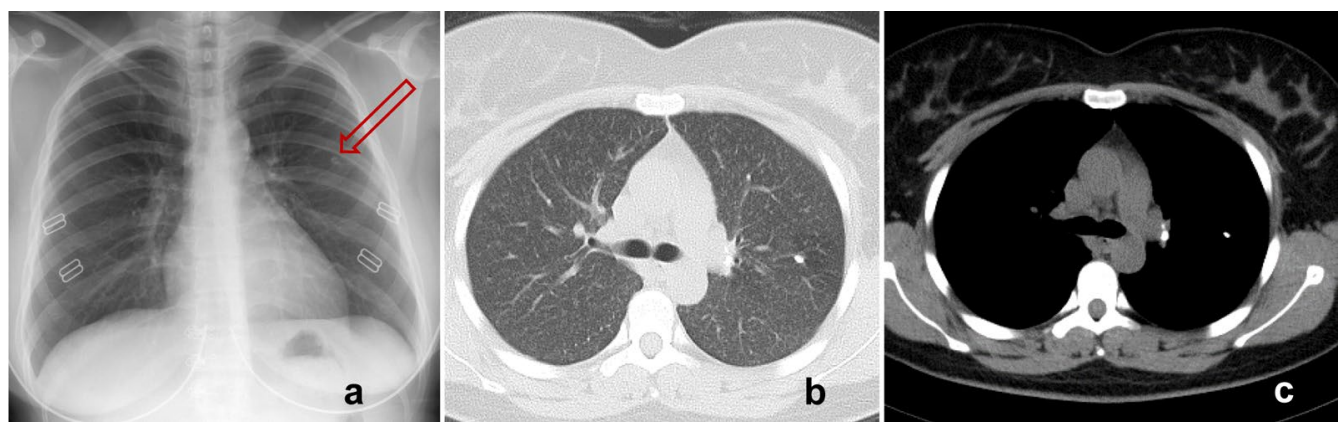

Fig. 2 Chest imaging in a 32-year-old infertile woman. a Posteroanterior chest radiograph showing a scarring nodule in the left upper lobe (arrow) at screening before IVF-ET. $\mathbf{b}$ Chest computed tomography showing multiple miliary nodules of uniform density, size, and distribution. $\mathbf{c}$ A scarring nodule in the left upper lobe and calcification of the left hilar lymph nodes. IVF-ET, in vitro fertilization and embryo transfer

\section{Outcomes of TB and pregnancy outcomes of the seven patients}

After the diagnosis of TB, four patients had spontaneous abortion, whereas three patients underwent induced abortion (Table 2). All seven patients recovered after anti-TB therapy. At follow-up, two patients achieved pregnancy after second IVF-ET.

\section{Discussion}

In this retrospective study, we identified seven cases of active TB during pregnancy from 62,755 cases of IVFET carried out at our hospital. All of these seven cases were diagnosed with acute miliary $\mathrm{TB}$, with two cases complicated by TB meningitis. Notably, signs of prior TB on CXR were detected in $11.4 \%$ of our study population $(7137 / 62,755)$, and six out of the seven patients with acute miliary TB had prior TB signs identified on CXR before IVF-ET.

Our data indicated that TB in pregnancy after IVF-ET mostly occurred during the first 8-12 weeks of pregnancy. Fever was the main symptom, and the time interval between fever onset and definitive diagnosis was 2-4 weeks or more. An important finding of our study was that all seven patients with active TB during pregnancy after IVF-ET developed hematogenous dissemination, which is the most serious condition of TB. Two out of the seven patients had tuberculous meningitis as a complication. This finding is consistent with the result of a previous report. We conducted a literature review on patients with TB during pregnancy after IVF-ET using the keywords "infertility" "in vitro fertilization and embryo transfer," "tuberculosis," and "pregnancy" to search for articles published from 1980 to 2019 in PubMed, MEDLINE, EMBASE, and Chinese Wanfang databases. Furthermore, we summarized 37 cases of TB during pregnancy after IVF-ET [10, 16-24] (Table 3). Addis et al. reported the first case in 1988 [10]. Since then, more cases have been described, the majority of which were from developing countries, [17-21, 24]. The results from our study and from these previous studies indicated that women with TB during pregnancy after IVF-ET were prone to hematogenous dissemination.

$\mathrm{TB}$ activation and dissemination may be related to latent infection, IVF-ET intervention, and immune dysregulation in pregnancy [25]. Studies have shown that estrogen, progesterone, and human chorionic gonadotropin have a direct inhibitory effect on T-cells [26, 27]. High estrogen levels are conducive to the proliferation of $M$. tuberculosis. Increased vascular permeability after pregnancy may also facilitate bacterial spread throughout the body, resulting in hematogenous dissemination [28]. The prognosis of miliary TB during pregnancy after IVFET was poor and may have caused the spontaneous abortion or may have resulted in premature delivery. More seriously, respiratory failure and even acute respiratory distress syndrome might occur in pregnant women [14, 16]. Furthermore, fetuses might suffer from intrauterine growth retardation or be stillborn due to hypoxia, or acquire infection via hematogenous dissemination or aspiration of contaminated amniotic fluid [11]. Moreover, those with miliary TB during pregnancy were less likely to achieve pregnancy, even with IVF-ET.

Identifying patients at high risk for TB activation should be an important evaluation before IVF-ET, especially in regions with a high TB burden. From our observation, we speculate that the coexistence of primary infertility, untreated prior pulmonary $\mathrm{TB}$, and fallopian tube obstruction may be a risk factor for active TB during an IVF-ET pregnancy. Signs of fibrotic scarring, calcified nodules, and/or pleural thickening on CXR indicate previous infection with M. tuberculosis [29-31]. In our series, among the 7137 patients who had "old TB" lesions on CXR, 


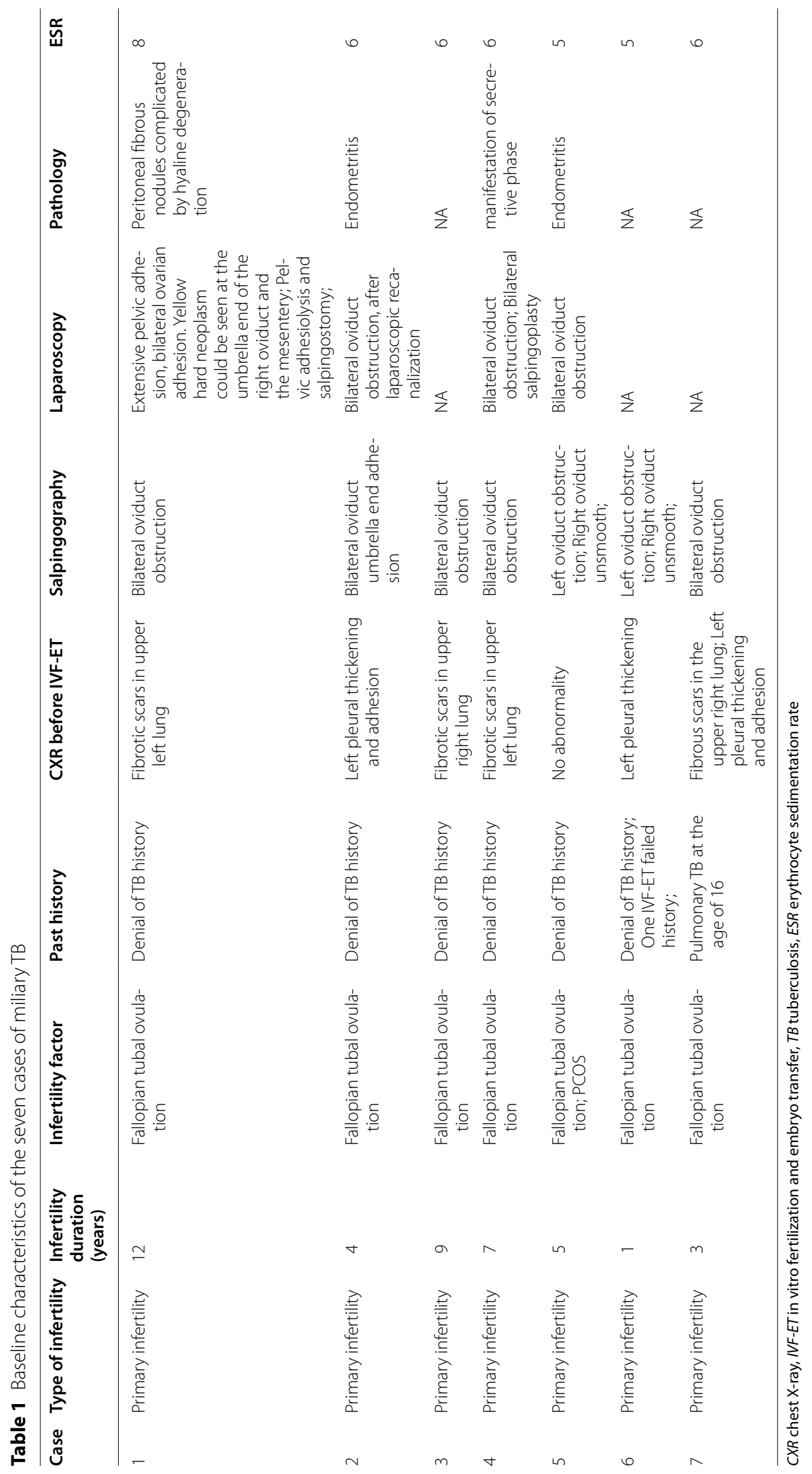




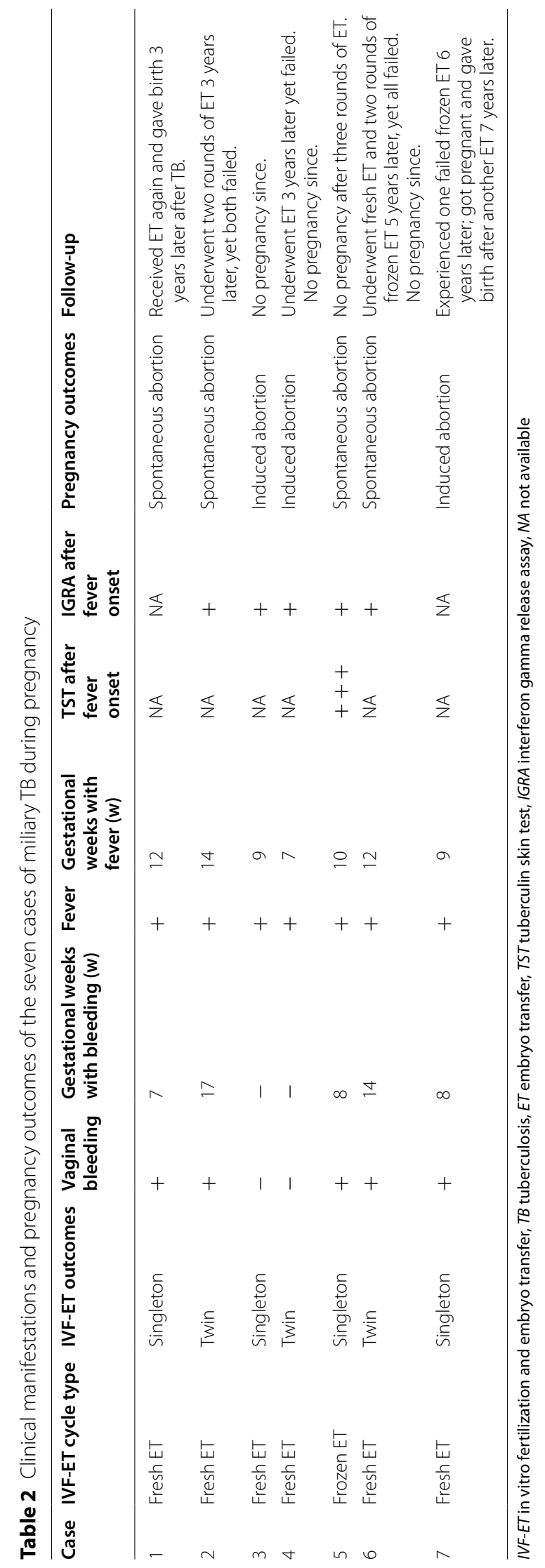




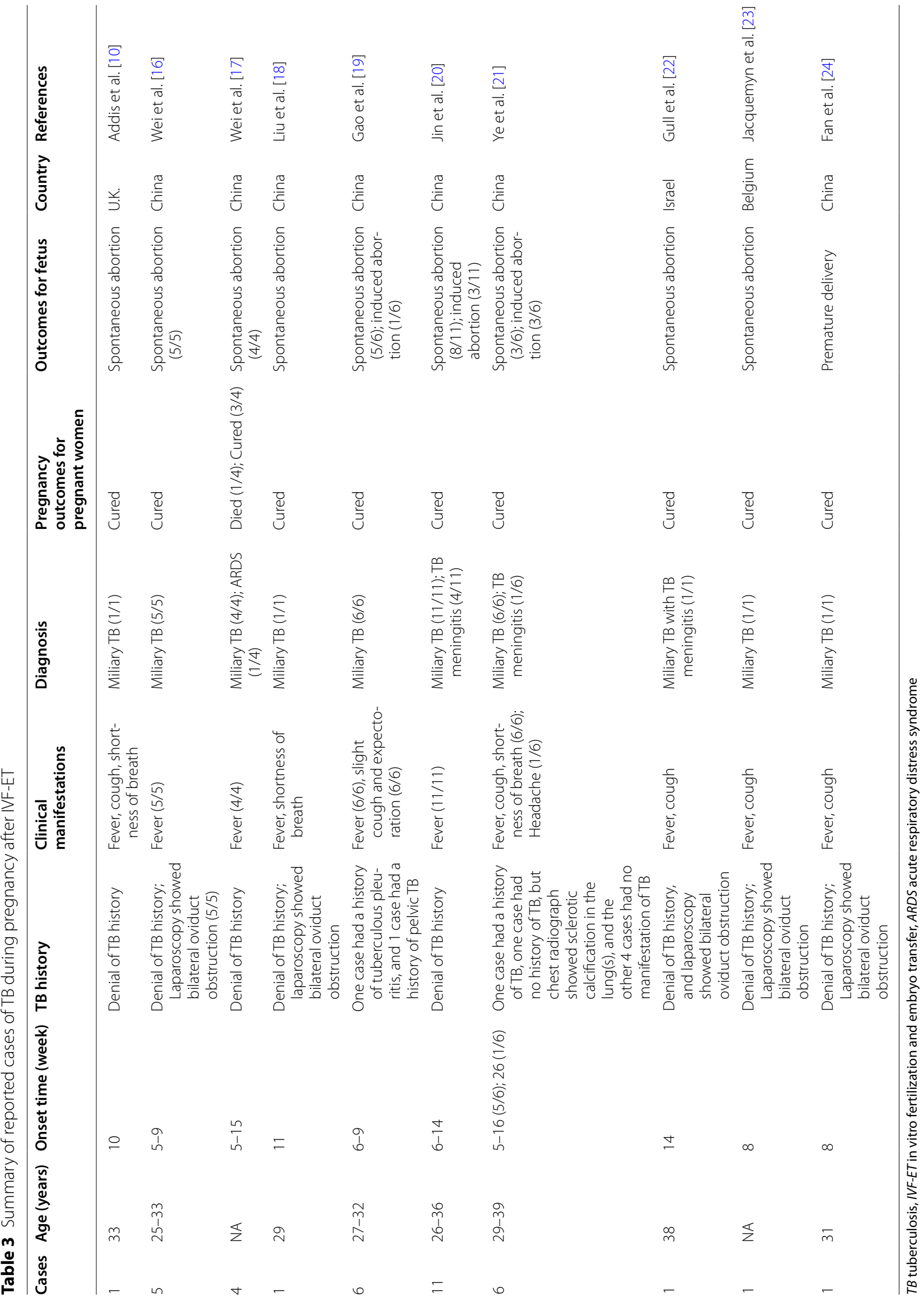


six developed miliary TB during pregnancy. Liu et al. reported a similar case in which untreated prior pulmonary $\mathrm{TB}$ developed into miliary $\mathrm{TB}$ during pregnancy [18]. Our previous study revealed that the clinical pregnancy and live birth rates were significantly lower in infertile patients with untreated prior pulmonary TB than in those without signs of prior TB, highlighting the adverse effects of TB in this specific patient population [32].

Genital TB (GTB) is a form of extrapulmonary TB and a major cause of primary infertility among women in TB-endemic countries [33], with a prevalence rate of $28.4 \%$ in our hospital, as observed in previous studies [34, 35]. GTB may cause fallopian tube obstruction, reduced endometrial receptivity, and ovarian dysfunction, leading to infertility. However, manifestations of GTB are nonspecific, and confirmation of diagnosis relies on invasive procedures. Our seven patients showed unilateral or bilateral oviduct obstruction, which suggested chronic infections such as GTB. Further studies are required to clarify whether the IGRA test and TST are necessary for the assessment of latent TB infection prior to IVF-ET and whether preventive anti-TB therapy can improve the pregnancy outcomes of infertile women with latent TB infection or untreated prior pulmonary TB on CXR. Moreover, screening for latent TB infection during pregnancy can provide an excellent opportunity for prevention.

Imaging plays a pivotal role in the diagnosis of pulmonary diseases, including ТВ [31, 36]. Clinical diagnosis of active TB in pregnant women is often delayed, which is largely attributable to the concern about radiation exposure from chest radiography. The IGRA test is an important diagnostic method for active TB detection and is safe for use during pregnancy [37-40]. Both the IGRA test and TST have a high consistency of $77.3-88.0 \%$ [39]. TheIGRA test has a high sensitivity of $100 \%$ and a moderate specificity of $80.0 \%$ for detectingactive TB during pregnancy [40], which are not affected by previous vaccination withbacillus Calmette-Guérin. Further studies on the use of the IGRA test for TB detectionduring pregnancy, particularly in high-risk patients from TB-endemic regions, are warranted.

Our study has some limitations. First, this was a single-center study; however, as the largest reproductive center in China, we perform more than 10,000 cycles of IVF-ET annually on women from all over the country. Therefore, the population in this study was representative. Second, we inquired whether active TB had occurred during pregnancy through telephone followup, which might have led to an underdiagnosis of the disease.

\section{Conclusions}

Acute miliary TB occurs in pregnant women after IVFET, particularly in those exhibiting signs of prior pulmonary TB on CXR. Patients with miliary TB have poor pregnancy outcomes. The coexistence of primary infertility, untreated prior pulmonary $\mathrm{TB}$, and fallopian tube obstruction is a high-risk factor for TB dissemination. Therefore, clinicians should be aware of the signs of TB before administering a course of IVF-ET treatment. Prospective studies are warranted to determine the incidence of and risk factors for reactive TB in infertile patients with prior pulmonary TB after IVF-ET and to clarify whether anti-TB therapy is beneficial for pregnancy outcomes in these patients.

\section{Abbreviations}

CT: Computed tomography; CXR: Chest X-ray; GTB: Genital TB; IGRA: Interferon gamma release assay; IVF-ET: In vitro fertilization and embryo transfer; TB: Tuberculosis; TST: Tuberculin skin test.

\section{Acknowledgements}

Not applicable.

\section{Authors' contributions}

$\mathrm{RL}$ and $\mathrm{YS}$ jointly conceived and directed this work, and $\mathrm{RL}$ is the leading corresponding author. XG, HC, and WC had full access to all data in the study and take responsibility for the integrity of the data and the accuracy of data analysis. XG, HC, WC, LZ, LC, WZ, DS, YW, and PL participated in the acquisition, analysis, or interpretation of the data. $X \mathrm{G}$ and $\mathrm{HC}$ wrote the report. $Y S$ and $\mathrm{RL}$ were involved in manuscript editing. The final version has been reviewed and approved by all authors. All authors read and approved the final manuscript.

\section{Funding}

This research was funded by the National Natural Science Foundation [No. 81400041 and 81871212] and the National Natural Science Foundation of China Youth Fund Project [No. 81400038]. The sponsors of the study had no role in study design, data collection, data analysis, data interpretation, or writing of the report. The corresponding author confirms that she had full access to all data in the study and had the final responsibility for the decision to submit for publication.

\section{Availability of data and materials}

The datasets used and/or analyzed during the current study are available from the corresponding author on reasonable request.

\section{Declarations}

\section{Ethics approval and consent to participate}

This study was approved by the Ethics Committee of Peking University Third Hospital [batch number: (2019)327-02]. The retrospective nature of the study resulted in a waiver regarding the signing of the informed consent form.

\section{Consent for publication}

Written informed consent was obtained from the seven patients for publication of their cases.

\section{Competing interests}

The authors declare that they have no competing interests.

\section{Author details}

'Department of Respiratory and Critical Care Medicine, Peking University Third Hospital, 49 N Garden Rd, Haidian District, Beijing 100191, China. ${ }^{2}$ Center for Reproductive Medicine, Department of Obstetrics and Gynecology, Peking University Third Hospital, 49 N Garden Rd, Haidian District, 100191 Beijing, 
China. ${ }^{3}$ Tuberculosis Department, Beijing Geriatric Hospital, 102699 Beijing China. ${ }^{4}$ Clinical Epidemiology Research Center, Peking University Third Hospital, 100191 Beijing, China. ${ }^{5}$ National Clinical Research Center for Obstetrics and Gynecology, 100191 Beijing, China. ${ }^{6}$ Key Laboratory of Assisted Reproduction (Peking University), Ministry of Education, 100191 Beijing, China. ${ }^{7}$ Beijing Key Laboratory of Reproductive Endocrinology and Assisted Reproductive Technology, 100191 Beijing, China.

Received: 19 August 2020 Accepted: 12 August 2021

Published online: 06 September 2021

\section{References}

1. World Health Organization. Global TB report. 2020. https://www.who.int/ teams/global-tuberculosis-programme/tb-reports. Accessed 3 Nov 2020

2. Sugarman J, Colvin C, Moran AC, Oxlade O. Tuberculosis in pregnancy: an estimate of the global burden of disease. Lancet Glob Health. 2014;2:e710-6.

3. Zumla A, Bates M, Mwaba P. The neglected global burden of tuberculosis in pregnancy. Lancet Glob Health. 2014;2:e675-6.

4. Sulis G, Pai M. Tuberculosis in pregnancy: a treacherous yet neglected issue. J Obstet Gynaecol Can. 2018:40:1003-5.

5. Bates M, Ahmed Y, Kapata N, Maeurer M, Mwaba P, Zumla A. Perspectives on tuberculosis in pregnancy. Int J Infect Dis. 2015:32:124-7.

6. Sharma SK, Mohan A, Sharma A. Miliary tuberculosis: a new look at an old foe. J Clin Tuberc Other Mycobact Dis. 2016;18:13-27.

7. Sharma SK, Mohan A. Miliary tuberculosis. Microbiol Spectr. 2017. https://doi. org/10.1128/microbiolspec.TNMI7-0013-2016.

8. Sobhy S, Babiker Z, Zamora J, Khan KS, Kunst H. Maternal and perinatal mortality and morbidity associated with tuberculosis during pregnancy and the postpartum period: a systematic review and meta-analysis. BJOG. 2017; 124:727-33.

9. Li Q, Song Y, Chen H, Xie L, Gao M, Ma L, et al. Retrospective analysis of 28 cases of tuberculosis in pregnant women in China. Sci Rep. 2019;9:15347.

10. Addis GM, Anthony GS, d'A Semple P, Miller AW. Miliary tuberculosis in an in-vitro fertilization pregnancy: a case report. Eur J Obstet Gynecol Reprod Biol. 1988:27:351-3.

11. Samedi V, Field SK, Al Awad E, Ratcliffe G, Yusuf K. Congenital tuberculosis in an extremely preterm infant conceived after in vitro fertilization: case report. BMC Pregnancy Childbirth. 2017;17:66.

12. Diseases Prevention and Control Department of China Health and Family Planning Commission. Guidelines for implementing the National Tuberculosis Control Program in China. Beijing: Peking Union Medical College Press; 2008.

13. Ray S, Talukdar A, Kundu S, Khanra D, Sonthalia N. Diagnosis and management of miliary tuberculosis: current state and future perspectives. Ther Clin Risk Manag. 2013;9:9-26

14. Deng W, Yu M, Ma H, Hu LA, Chen G, Wang Y, et al. Predictors and outcome of patients with acute respiratory distress syndrome caused by miliary tuberculosis: a retrospective study in Chongqing, China. BMC Infect Dis. 2012:12:121.

15. Wang $\mathrm{H}, \mathrm{GaO} H$, Chi H, Zeng $L$, Xiao W, Wang $Y$, et al. Effect of levothyroxine on miscarriage among women with normal thyroid function and thyroid autoimmunity undergoing in vitro fertilization and embryo transfer: a randomized clinical trial. JAMA. 2017:318:2190-8.

16. Wei $Y$, Wen $E$, Zhao $Y Y$, Wang LN, Qiao J. In vitro fertilization and embryo transplantation pregnancy complicated by acute miliary tuberculosis in five cases. Chin J Perinat Med. 2010;13:324-6 In Chinese.

17. Wei $Y, Z$ Zhao $Y Y$, Wang YQ. The clinic analysis of four cases of pregnancy of women with miliary tuberculosis after in vitro fertilization and embryo transfer. Int J Gynecol Obstet. 2009;107:545.

18. Liu HB, Zhao L. Miliary tuberculosis after in vitro fertilization and embryo transplantation. Afr Health Sci. 2015;15:701-4.

19. Gao HJ, Hu YJ, Zhu YM, Huang HF. Retrospective analysis of 6 cases of miliary tuberculosis in pregnant women after in vitro fertilization and embryo transplantation. Chin J Tuberc Respir Dis. 2007;30:848-50 In Chinese with English abstract.

20. Jin C, Mi Z, Sun LF. Clinical characteristics of pulmonary tuberculosis in pregnant women after in vitro fertilization embryo transfer. Chin J Clin Infect Dis. 2014;7:137-9 In Chinese with English abstract.
21. Ye R, Wang C, Zhao L, Wu X, Gao Y, Liu H. Characteristics of miliary tuberculosis in pregnant women after in vitro fertilisation and embryo transplantation. Int J Tuberc Lung Dis. 2019;23:136-9.

22. Gull I, Peyser MR, Yaron Y, Jaffa AJ, Amit A, Lessing JB. The effect of an in-vitro fertilization pregnancy on a woman with genital tuberculosis. Hum Reprod. 1995; 10:3052-4

23. Jacquemyn Y, Van Casteren C, Luijks M, Colpaert C. Disseminated tuberculosis in pregnancy unknown to doctors in Western Europe case presentation:'part of the routine study in infertility'. BMJ Case Rep. 2012 2012:bcr2012006227.

24. Fan XL, Chen ZJ, Li CY, Ma L. Analysis of a case of miliary tuberculosis in both lungs and premature delivery after in vitro fertilization and embryo transplantation pregnancy. Chin J Obstet Gynecol. 2003;38:637-8 In Chinese.

25. Singh N, Perfect JR. Immune reconstitution syndrome and exacerbation of infections after pregnancy. Clin Infect Dis. 2007;45:1192-9.

26. Aghaeepour N, Ganio EA, Mcilwain D, Tsai AS, Tingle M, Van Gassen S, et al. An immune clock of human pregnancy. Sci Immunol. 2017;2:eaan2946.

27. Saito S, Nakashima A, Shima T, Ito M. Th1/Th2/Th17 and regulatory T-cell paradigm in pregnancy. Am J Reprod Immunol. 2010;63:601-10.

28. Yu N, Yang J, Guo Y, Fang J, Yin T, Luo J, et al. Intrauterine administration of peripheral blood mononuclear cells (PBMCs) improves endometrial receptivity in mice with embryonic implantation dysfunction. Am J Reprod Immunol. 2014;71:24-33.

29. Uzorka JW, Kroft LJM, Bakker JA, van Zwet EW, Huisman E, Prins C, et al. Abnormalities suggestive of latent tuberculosis infection on chest radiography; how specific are they? J Clin Tuberc Other Mycobact Dis. 2019;15:100089.

30. Uzorka JW, Wallinga J, Kroft LJM, OttenhoffTHM, Arend SM. Radiological signs of latent tuberculosis on chest radiography: a systematic review and meta-analysis. Open Forum Infect Dis. 2019;6:ofz313.

31. Nachiappan AC, Rahbar K, Shi X, Guy ES, Barbosa EJM Jr, Shroff GS, et al. Pulmonary tuberculosis: role of radiology in diagnosis and management. Radiographics. 2017:37:52-72.

32. Gai $X Y$, Chi HB, Zeng L, Cao WL, Chen LX, Zhang C, et al. Untreated prior pulmonary tuberculosis adversely affects pregnancy outcomes in infertile women undergoing in vitro fertilization and embryo transfer: a large retrospective cohort study. Biomed Environ Sci. 2021;34:130-8.

33. Bhanothu V, Theophilus JP, Reddy PK, Rozati R. Occurrence of female genital tuberculosis among infertile women: a study from a tertiary maternal health care research centre in South India. Eur J Clin Microbiol Infect Dis. 2014;33:1937-49

34. Zhang LZ, Wei ZX, Liu P. Factors affecting the clinical pregnancy rate in an in vitro fertilization and embryo transfer program. Chin J Obstet Gynecol. 1998;33:727-30 In Chinese with English abstract.

35. Li MM, Li R. Progress in diagnosis and treatment of female genital tuberculosis induces infertility. Chin J Obstet Gynecol. 2015:50:954-6 In Chinese.

36. Razek AAKA, El Badrawy MK, Alnaghy E. Interstitial lung fibrosis imaging reporting and data system: what radiologist wants to know? J Comput Assist Tomogr. 2020:44:656-66.

37. Gupta RK, Kunst H, Lipman M, Noursadeghi M, Jackson C, Southern J, et al. Evaluation of QuantiFERON-TB Gold Plus for predicting incident tuberculosis among recent contacts: a prospective cohort study. Ann Am Thorac Soc 2020;17:646-50.

38. Abubakar I, Drobniewski F, Southern J, Sitch AJ, Jackson C, Lipman M, et al. Prognostic value of interferon-g release assays and tuberculin skin test in predicting the development of active tuberculosis (UK PREDICT TB): a prospective cohort study. Lancet Infect Dis. 2018;18:1077-87.

39. Lighter-Fisher J, Surette AM. Performance of an interferon-gamma release assay to diagnose latent tuberculosis infection during pregnancy. Obstet Gynecol. 2012;119:1088-95.

40. Chen Q, Guo X, Wang X, Wang M. T-SPOT.TB in detection of active tuberculosis during pregnancy: a retrospective study in China. Med Sci Monit. 2016;22:57-60.

\section{Publisher's Note}

Springer Nature remains neutral with regard to jurisdictional claims in published maps and institutional affiliations. 